\section{Buchrezension zu: Umweltmikrobiologie}

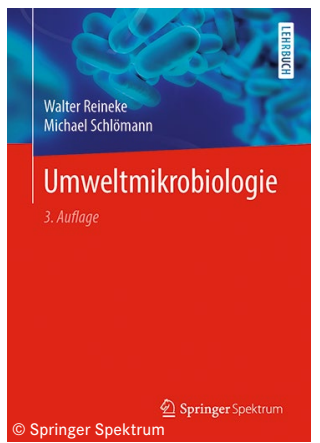

\section{Umweltmikrobiologie}

Walter Reineke und Michael Schlömann

647 S., 335 Abb., Springer, 2020.

3. Aufl., SC, 44,99€

ISBN: 9783662596548

Auch als E-Book erhältlich

DOI: $10.1007 / \mathrm{s} 12268-021-1522-8$

(C) Der Autor 2021

Vielfältig, effektiv und oft unsichtbar: Mikroorganismen sind überall auf unserem Planeten. Sie treiben den Kohlenstoff- und andere Elementkreisläufe an, bilden eine ähnlich große Biomasse wie Pflanzen, und in nur einer Handvoll Erde leben mehr Bakterien als es Menschen je gegeben hat. Das Lehrbuch ,Umweltmikrobiologie“ ist eine exzellente Einführung in dieses faszinierende Reich mikrobieller Gemeinschaften und deren Rolle bei Umweltprozessen.

Die Autoren verstehen Umweltmikrobiologie dabei auch als angewandte Wissenschaft zur Vermeidung und Beseitigung anthropogener Umweltbelastungen. Geschrieben ist es als leicht lesbares Lehrbuch für Studierende der Lebens-, Umwelt- und Umweltingenieurswissenschaften. Gleichwohl ist es auch ein unverzichtbarer Begleiter für alle, die sich für mikrobielle Ökosysteme und deren umweltbiotechnologische Anwendungen interessieren, z. B. beim Schadstoffabbau oder bei der Reinigung verschmutzter Abwässer.

Die 19 gut strukturierten Kapitel des Buchs befassen sich mit - der Rolle von Mikroorganismen in der Biosphäre und globalen Stoffkreisläufen,
- den wichtigsten mikrobiellen Transformationsprozessen von Schadstoffen,

- den mikrobiellen Anpassungsstrategien an Umweltlebensräume,

- Nachweismethoden der Diversität und Aktivität mikrobieller Gemeinschaften und

- Fragen des Umweltschutzes sowie biologischer Sanierungs- und Abfallbehandlungstechniken.

Jedes Kapitel kann ohne weiteres für sich allein gelesen werden und wird für Lernende durch nützliche Verständnisfragen und einen kleinen Literaturteil ergänzt.

Aufgrund der klaren Lesbarkeit, der interdisziplinären Themenauswahl und eines luziden Schlusskapitels, das die Lesenden zum Nachdenken über Nachhaltigkeit und die Relevanz mikrobieller Prozesse für das tägliche Leben anregt, ist diese dritte - nun auch online erhältliche - Auflage eine zu empfehlende Reise durch die spannende Welt der Umweltmikrobiologie. Eigentlich schade, dass das Buch nur in deutscher Sprache und mit meist schwarzweißen Abbildungen erhältlich ist.

Lukas Y. Wick Helmholtz Centre for Environmental Research - UFZ, Leipzig lukas.wick@ufz.de

Diese Rezension erscheint Open Access. *
* Funding note: Open Access funding enabled and organized by Projekt DEAL. Open Access: Dieser Artikel wird unter der Creative Commons Namensnennung 4.0 International Lizenz veröffentlicht, welche die Nutzung, Vervielfältigung, Bearbeitung, Verbreitung und Wiedergabe in jeglichem Medium und Format erlaubt, sofern Sie den/die ursprünglichen Autor(en) und die Quelle ordnungsgemäß nennen, einen Link zur Creative Commons Lizenz beifügen und angeben, ob Änderungen vorgenommen wurden. Die in diesem Artikel enthaltenen Bilder und sonstiges Drittmaterial unterliegen ebenfalls der genannten Creative Commons Lizenz, sofern sich aus der Abbildungslegende nichts anderes ergibt. Sofern das betreffende Material nicht unter der genannten Creative Commons Lizenz steht und die betreffende Handlung nicht nach gesetzlichen Vorschriften erlaubt ist, ist für die oben aufgeführten Weiterverwendungen des Materials die Einwilligung des jeweiligen Rechteinhabers einzuholen. Weitere Details zur Lizenz entnehmen Sie bitte der Lizenzinformation auf

http://creativecommons.org/licenses/ by $/ 4.0 /$ deed.de. 